\title{
Memorandum zur Forschungsförderung in der Prävention - alter Wein in neuen Schläuchen?
}

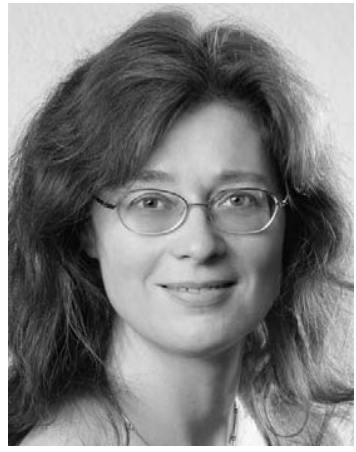

Prof. Dr. Ulla Walter

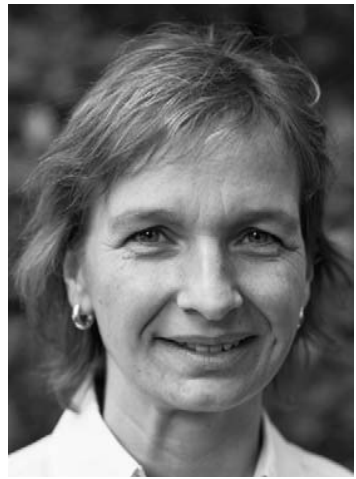

Dr. Silke Pawils, Dipl.-Psych.
Memoranden haben, so scheint es, Konjunktur. Sie deuten auf Missstände hin, fassen konsentierte Grundsätze zusammen und bieten Empfehlungen als Lösungsansatz an. Ein Memorandum ist, so der Blick in das etymologische Wörterbuch nach Pfeifer, eine Denkschrift. Dem lateinischen Wortsinn nach möchte ein Memorandum „in Erinnerung bringen, was erinnernswert ist“. Nun also noch ein Memorandum, und dieses zur Prävention. Ist nicht schon alles gesagt?

Dass ein durchgreifender politischer Gestaltungswille zur nachhaltigen Umsetzung und Förderung der Prävention und Gesundheitsförderung in Deutschland fehlt, wurde mehrfach beklagt, die seit 20 Jahren gleichbleibend geringen Ausgaben um 4\% der Gesundheitsausgaben (www.gbe.bund.de) werden als Stagnation der Bemühungen moniert, und die systematische Integration in die gesundheitsbezogene Versorgung [1] wird seit Jahren angemahnt.

Parallel zur politischen Umsetzung ist eine grundlagen- und anwendungsorientierte Forschung in der Prävention und Gesundheitsförderung notwendig, um (a) die theoretischen Grundlagen zu überprüfen und weiterzuentwickeln, (b) Bedarfe und Zielgruppen zu identifizieren, (c) Interventionen zielgerichtet zu planen, umzusetzen und ihre Effekte zu bewerten sowie (d) erfolgreiche Maßnahmen qualitätsgesichert zu implementieren und in andere Kontexte zu transferieren. Die WHO definierte 2005 die Förderung von grundlagen- und anwendungsorientierter Forschung mit einem Fokus auf der Entwicklung wirksamer Strategien und ihre Integration in die Versorgungspraxis als Ziel zur Prävention chronischer Erkrankungen [2].

Prävention und Gesundheitsförderung, die wirkt, basiert dabei immer auf Forschungserkenntnissen diverser Disziplinen. Das jüngst vorgelegte Memorandum zur sozial-ökologischen Forschung in Deutschland [3] verweist auf die Notwendigkeit der transdisziplinären Forschung, die sich auszeichnet durch eine „Orientierung an gesellschaftlichen Problemen, die Integration unterschiedlicher wissenschaftlicher und praktischer Wissensformen sowie die Produktion sozial robusten Wissens - ein Wissen, das partizipativ erzeugt und bewertet ist" [3, S. 4].

Dieses gilt auch für die Prävention und Gesundheitsförderung. In dem Forschungsschwerpunkt „Präventionsforschung“ des Bundesministeriums für Bildung und Forschung (BMBF), der erstmals die primäre Prävention und Gesundheitsförderung explizit förderte, bildete die enge Zusammenbindung zwischen Wissenschaft und Praxis eine zentrale Voraussetzung der Förderung.
Lebensphasen und -lagenbezogen setzte der Förderschwerpunkt an Zielgruppen wie Kinder und Jugendliche, ältere Menschen sowie sozial benachteiligte Menschen an. Gegenstand der inhaltlichen Förderung waren eine Überprüfung der Wirksamkeit und Qualitätssicherung bereits anstehender oder neu konzipierter Maßnahmen sowie die Entwicklung und Erprobung neuer Zugangswege zu einer Zielgruppe oder einer Lebenswelt. Die Projekte sollten zudem einen innovativen Beitrag zur Weiterentwicklung methodischer Kompetenzen leisten.

In 4 Förderphasen wurden im Zeitraum von 2004-2012 rund 60 Projekte mit einem Gesamtvolumen von 20,05 Mio. Euro unterstützt. Eingebunden waren über 50 wissenschaftliche Einrichtungen und 170 sogenannte Praxispartner, Akteure in den Bereichen Gesundheit, Bildung, Arbeit und Soziales auf kommunaler, Landesund Bundesebene.

In dieser Zusammenbindung von Wissenschaft und Praxis wurden über eine kontinuierliche Auseinandersetzung und Reflexion in unterschiedlichsten Kontexten vielfältige Erfahrungen im Verlauf des Forschungsprozesses auf beiden Seiten gewonnen. Dieses implizite und für die Weiterentwicklung von Prävention und Gesundheitsförderung insgesamt erforderliche Wissen wurde in einem aufwendigen partizipativen Prozess zusammengeführt und in dem nun vorliegenden Memorandum zur Forschungsförderung Prävention gebündelt.

Angeregt und unterstützt wurde dieser Prozess durch das Meta-Projekt „Kooperation für nachhaltige Präventionsforschung“(KNP), das im letzten Drittel des Förderschwerpunkts eingerichtet wurde. Das Projekt soll die Distribution wesentlicher Erkenntnisse unterstützen, die Vernetzung zwischen Wissenschaft, Praxis und Politik fördern und Wissensmanagement im Bereich der Präventionsforschung nutzen [4].

Mit dem Memorandum zur Forschungsförderung sollen in erster Linie politischen Entscheidungsträgern Hinweise zur Optimierung der Forschungsförderung im Bereich der Primärprävention und Gesundheitsförderung gegeben werden, um den begonnenen Forschungsprozess konstruktiv weiterzuführen.

Ausgehend von den Erfahrungen mit der bisherigen Forschungsförderung werden daher Empfehlungen zur künftigen Forschungsförderung gegeben, die in einem breiten Abstimmungsprozess mit allen Projektverantwortlichen des BMBFFörderschwerpunkts, 13 relevanten Fachgesellschaften und den Beiräten der Bundeszentrale 
für gesundheitliche Aufklärung (BZgA) und von KNP anerkannt wurden.

Die inhaltlichen Empfehlungen umfassen:

- Rahmenbedingungen für anwendungsorientierte Forschung wie beispielsweise Anpassung der Projektlaufzeiten, Zweistufigkeit des Antragsverfahrens, Inhalte der Kooperation Wissenschaft - Praxis, Monitoring-Phase, Publikationsphase in der Förderphase, Transfer-Anschlussförderphase und

- Rahmenbedingungen für grundlagenorientierte Forschung wie beispielsweise von Verfahren wie Meta-Analysen und systematischen Reviews, aber auch Strukturbildung durch Graduiertenkollegs und der Einbezug von Gutachter/innen mit Expertise in Primärprävention und Gesundheitsförderung.

Diese Empfehlungen erscheinen in Kreisen praxisbezogener Forschung hinlänglich bekannt. Und dennoch: Bei breiter Akzeptanz aus allen relevanten Fachgesellschaften und mit hoher Praktikabilität liegen die Empfehlungen nun für den politischen
Diskurs erstmalig aufbereitet vor. Wissenschaft und Praxispartner hat dieser Prozess vorangebracht. Nun liegt es in der Hand der politischen Entscheidungsträger, die gewonnenen Erkenntnisse aufzunehmen und das bereitete Feld fruchtbar zu nutzen.

\section{Literatur}

1 Sachverständigenrat zur Begutachtung der Entwicklung im Gesundheitswesen. Koordination und Integration - Gesundheitsversorgung in einer Gesellschaft des längeren Lebens. Sondergutachten 2009. Baden-Baden: Nomos; 2010

2 World Health Organization. Preventing chronic diseases - a vital investment. WHO global report; Genf: 2005

3 Verstehen - Bewerten - Gestalten. Transdisziplinäres Wissen für eine nachhaltige Gesellschaft. Memorandum zur Weiterentwicklung der sozial-ökologischen Forschung in Deutschland. 2012; http://www. sozial-oekologische-forschung.org/de/1651.php

4 Walter U, Plaumann M, Dubben S et al. Effectiveness, quality and transfer: further development of disease prevention and health promotion through research. DMW - Deutsche medizinische Wochenschrift 2011; 136: 1488-1493 